\title{
A new vector system for targeted integration and overexpression of genes in the crop pathogen Fusarium solani
}

\author{
Mikkel Rank Nielsen 1* (D, Anna Karolina Rilana Holzwarth', Emmett Brew', Natalia Chrapkova1, \\ Samba Evelyne Kabemba Kaniki ${ }^{1}$, Kenneth Kastaniegaard ${ }^{2}$, Trine Sørensen², Klaus Ringsborg Westphal ${ }^{2}$, \\ Reinhard Wimmer ${ }^{2}$, Teis Esben Sondergaard ${ }^{2}$ and Jens Laurids Sørensen ${ }^{{ }^{*}}$
}

\begin{abstract}
Background: Besides their ability to produce several interesting bioactive secondary metabolites, members of the Fusarium solani species complex comprise important pathogens of plants and humans. One of the major obstacles in understanding the biology of this species complex is the lack of efficient molecular tools for genetic manipulation.

Results: To remove this obstacle we here report the development of a reliable system where the vectors are generated through yeast recombinational cloning and inserted into a specific site in F. solani through Agrobacterium tumefaciens-mediated transformation. As proof-of-concept, the enhanced yellow fluorescent protein (eYFP) was inserted in a non-coding genomic position of $F$. solani and subsequent analyses showed that the resulting transformants were fluorescent on all tested media. In addition, we cloned and overexpressed the $\mathrm{Zn}(\mathrm{II})_{2} \mathrm{Cys}_{6}$ transcriptional factor fsr 6 controlling mycelial pigmentation. A transformant displayed deep red/purple pigmentation stemming from bostrycoidin and javanicin.

Conclusion: By creating streamlined plasmid construction and fungal transformation systems, we are now able to express genes in the crop pathogen F. solani in a reliable and fast manner. As a case study, we targeted and activated the fusarubin (PKS3: $f s r)$ gene cluster, which is the first case study of secondary metabolites being directly associated with the responsible gene cluster in F. solani via targeted activation. The system provides an approach that in the future can be used by the community to understand the biochemistry and genetics of the Fusarium solani species complex, and is obtainable from Addgene catalog \#133094.
\end{abstract}

Keywords: Fusarium, Transformation, Heterologous expression, Fluorescence, Agrobacterium tumefaciens-mediated transformation, Secondary metabolites, Polyketides, Fusarubin, Bostrycoidin

\section{Background}

The Fusarium solani species complex (FSSC) is one of the most widespread fungal groups, which collectively is capable of causing disease in both plants and humans $[1,2]$. More than 50 species within the FSSC have been described $[1,3,4]$ and the number continuous to increase as new species are constantly identified [5], primarily

\footnotetext{
*Correspondence: mrn@bio.aau.dk; jls@bio.aau.dk

${ }^{1}$ Department of Chemistry and Bioscience, Aalborg University, Niels

Bohrs Vej 8, 6700 Esbjerg, Denmark

Full list of author information is available at the end of the article
}

based on molecular analyses. Members of the species complex are important plant pathogens of more than 100 agricultural crops where they can cause vascular wilt or root rot [6]. Individual species are often associated with only one or a few hosts and consequently plant pathogenic populations of $F$. solani have been further subdivided into formae speciales [7-9], although this view has been challenged recently [10]. The variation within the species complex is also reflected within sexual replication, where both heterothallic, homothallic and mitosporic species have been identified [3]. 
Several bioactive secondary metabolites have been identified in FSSC, including cyclosporin, gibepyrone A, lucilactaene, sansalvamide and the pigments fusarubin, javanicin and bostrycoidin [11]. Analyses of the genome of the first sequenced FSSC strain (77-13-4; FGSC 9596) revealed the presence of 13 polyketide synthases (PKSs) and 13 non-ribosomal peptide synthetases (NRPSs) [2] and subsequent orthology studies have linked fusarubin, javanicin, bostrycoidin (PKS3; fsr), gibepyrone A (PKS8, Gpy1), lucilactaene (PKS10) to their responsible gene clusters [12-17].

In the past, one of the obstacles in genetic editing of $F$. solani is the sparse reports of successful transformation protocols [18-20]. We recently overcame this obstacle through optimization of a protocol for Agrobacterium tumefaciens-mediated transformation (ATMT), which was used to identify the gene cluster behind biosynthesis of sansalvamide [21]. A second possible bottleneck in the transformation process can be construction of the ATMT vectors, which usually rely on correct cloning of the vector backbone containing genes for bacterial replication and selection together with two homologues recombination sequences and the integration cassette containing a selection marker and the overexpression system. Assembling these four fragments can be obtained through enzyme based methods, including Xi, In-Fusion, Gateway and USER Friendly cloning techniques [22]. The cloning process can be accelerated and made more cost-effective by in vivo homologous recombinational cloning in Saccharomyces cerevisiae where amplified DNA fragments are cloned guided by overlapping sequences of down to 15 bp [23, 24]. The combination of yeast based cloning and ATMT has developed for Aspergillus fumigatus in a time and labor reducing method [25]. The aim of the present study was to adapt this approach to F. solani to develop a system that allow easy generation of transformation vectors for overexpression of target genes from an active locus on the chromosome. As proof-of-concept, we chose the enhanced yellow fluorescent protein (eYFP) while the internal regulator of the $P K S 3$ gene cluster was targeted to demonstrate the usefulness in genetic engineering of biosynthetic gene clusters.

\section{Methods}

\section{Strains, media and conditions}

Fusarium solani mating population IV (77-13-4; FGSC 9596, Fungal Genetics Stock Center) was used as model for the transformation. The strain was maintained on Czapek Dox $(\mathrm{Cz})$ agar medium or potato dextrose agar (PDA) medium [26] during the experiments. Plasmid assembly was performed in Saccharomyces cerevisiae
BY4743 (Euroscarf Y20000, [27]), which was maintained on yeast extract peptone dextrose (YPD) medium and selected on yeast synthetic dropout medium without uracil (SC-U; Sigma-Aldrich, St. Louis, MO, USA; Y1501). Escherichia coli DH5 $\alpha$ was used for yeast-plasmid recovery and propagation. Transformed cells were grown and selected on solid (2\% agar) or liquid Luria-Bertani (LB, Lennox) medium supplemented with $50 \mu \mathrm{g} / \mathrm{mL}$ kanamycin at $37^{\circ} \mathrm{C}$. Agrobacterium tumefaciens AGL-1 was used for transformation of $F$. solani. The strain was grown on LB medium supplemented with $100 \mu \mathrm{g} / \mathrm{mL}$ rifampicin as well as $50 \mu \mathrm{g} / \mathrm{mL}$ kanamycin for selecting transformants carrying the assembled plasmid.

\section{Construction of pSHUT4-eYFP plasmid}

Four PCR fragments were required to generate the plasmid for constitutive expression of the eYFP gene from an active position on the genome. This approach was inspired from F. graminearum, where the aurofusarin cluster specific transcription factor aurR1 was recently overexpressed in a locus close to the $\beta$-Tubulin gene [28]. All PCRs were performed using Phusion Hot Start II DNA Polymerase (Thermo Fisher Scientific), using the supplied reagents and manufacturer's guidelines. Prior to the experiments, eYFP from the EarleyGate 104-vector [29] had been cloned into p-UGOTL-TEF1 $\alpha$-TF [30] to generate an expression cassette comprising the nptII fungal selection marker together with the constitutive translation elongation factor $1 \alpha$ (TEF-1 $\alpha$ ) promoter, eYFP and NOS terminator. This cassette was amplified using primers D094+D095 (see Additional file 1 for all primers in this study). For guiding the homologous integration in F. solani, were two fragments amplified from genomic DNA using primers D090 + D091 and D092 + D093. Specifically, were the two integration border sites amplified from a non-coding region between the genes NECHADRAFT_103550 (Conserved, hypothetical Fusarium protein) and NECHADRAFT_91300 (Putative ATP-binding ABC transporter), found just upstream from NECHADRAFT_66759 ( $\beta$-Tubulin). Finally, a plasmid backbone was PCR amplified from in-house vector pSHUT3 [31] with primers C094+C095. This vector is based on U-GOAL [32] and comprise the bacterial elements $k a n^{\mathrm{R}}$, IncP, trfA to which the yeast auxotrophic selection marker URA3 from pYES2 (Invitrogen) and replication origin CEN6/ARSH4 from pRS315 (ATCC ${ }^{\circledR} 77144$ ) [33] were added. Correct assembly of the plasmid was guided by adding 15 bp homology tails to all primers yielding a 10.460 bp plasmid dubbed pSHUT4eYFP (Fig. 1). The four PCR products were visualized by gel electrophoresis on $1 \%$ agarose gels and subsequently 


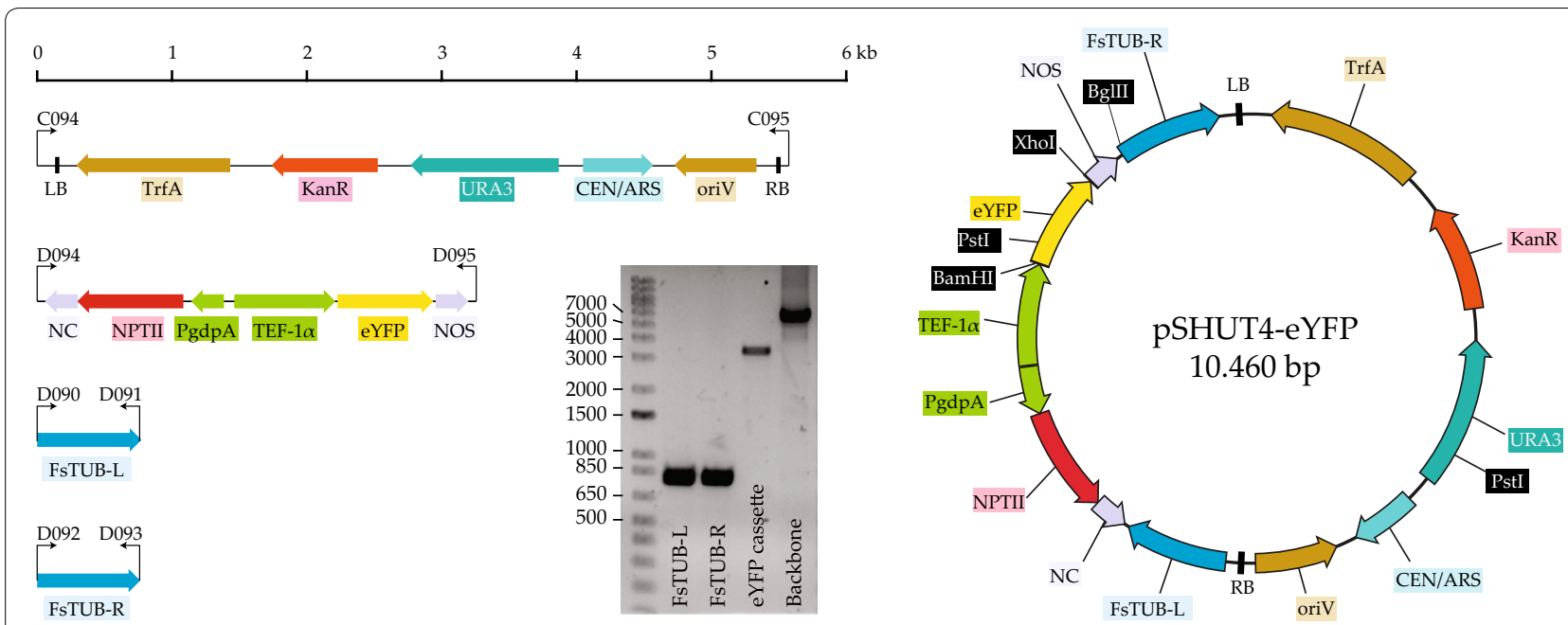

Fig. 1 Illustration and an actual agarose of the four PCR fragments, which are subsequently assembled into pSHUT4-eYFP via yeast recombinational assembly

cleaned by the QIAquick PCR purification kit (Qiagen). At least $200 \mathrm{fmol}$ of each of the four purified PCR fragments were transformed into S. cerevisiae by the lithium acetate/single-stranded carrier DNA/PEG method [34]. As controls, subsequent transformation reactions were set up where one of the four PCR fragments was omitted from the transformation mix and substituted with water. Yeast transformants were selected on solid SC-U and grown for 2 days at $30{ }^{\circ} \mathrm{C}$. Colonies were streaked on new $\mathrm{SC}-\mathrm{U}$ plates from where the plasmids were isolated from fresh yeast cells as previously described [35]. The isolated plasmids were electroporated into competent E. coli DH5 $\alpha$ cells using the BioRad Micropulser Electroporation Apparatus, following the manufacturer's instructions, and selected cells on solid LB medium with kanamycin. Assembled plasmid constructs were propagated and isolated from E. coli cultures using the QIAprep Spin Miniprep Kit (Qiagen). Sequencing of constructs was performed at Eurofins genomics (Ebersberg, Germany).

\section{Generation and transformation of $F$. solani macroconidia}

Five agar plugs from a 5-day-old $F$. solani culture on Czapek Dox agar medium was added to $70 \mathrm{~mL}$ CMC medium $[15 \mathrm{~g} / \mathrm{L}$ carboxymethyl-cellulose sodium salt (C4888; Sigma-Aldrich) $1 \mathrm{~g} / \mathrm{L} \mathrm{NH}_{4} \mathrm{NO}_{3}, 1 \mathrm{~g} / \mathrm{L} \mathrm{KH}_{2} \mathrm{PO}_{4}$, $0.5 \mathrm{~g} / \mathrm{L} \mathrm{MgSO}_{4} \cdot 7 \mathrm{H}_{2} \mathrm{O}, 1 \mathrm{~g} / \mathrm{L}$ Bacto yeast extract (Becton, Dickinson and Company, Sparks, MD, USA] [36] in a $250 \mathrm{~mL}$ baffled flask. The culture was incubated in the dark for 5 days at $20^{\circ} \mathrm{C}$ with $100 \mathrm{rpm}$. The medium was filtered through a sterilized syringe lightly packed with glass wool before the flow through was centrifuged at $5000 \mathrm{~g}$ for $40 \mathrm{~min}$ at $5{ }^{\circ} \mathrm{C}$. The supernatant was carefully discarded and the pellet resuspended in $10 \mathrm{~mL}$ cold sterile $\mathrm{H}_{2} \mathrm{O}$. This step was repeated twice with centrifugation for $20 \mathrm{~min}$, before the macroconidia were ready for transformation.

The F. solani macroconidia were transformed with pSHUT4-eYFP using A. tumefaciens AGL1 as previously described [21]. The transformation mixture containing $A$. tumefaciens and $F$. solani was spread onto induction medium plates containing a black filter paper (Frisenette, Denmark, 140.090). Positive controls included plates with and without filters plated with either plasmid carrying A. tumefaciens or macroconidia. After incubation at $22{ }^{\circ} \mathrm{C}$ for 3 days, the filters were moved to V8 agar plates [37] containing $150 \mu \mathrm{g} / \mathrm{mL}$ G418 (Gibco) and $300 \mu \mathrm{g} / \mathrm{mL}$ cefoxitin sodium (Sigma-Aldrich). After 7 days, the filters were moved to new V8 agar plates containing $150 \mu \mathrm{g} / \mathrm{mL}$ G418 where colonies started to emerge after $1-5$ days. Single colonies were transferred to potato dextrose agar (PDA) [26] plates containing $200 \mu \mathrm{g} / \mathrm{mL}$ G418. All incubation steps were carried out in darkness.

\section{Verification of transformants by PCR and sequencing}

Colony PCR was routinely used to screen isolated mutants with primers hybridizing outside integration locus and inside the T-DNA cassette. A minute amount of 2-3 day old hyphal mycelium was picked with a sterile toothpick and submerged in $300 \mu \mathrm{L}$ fungal lysis buffer [0.2 M NaCl, 0.1\% Triton-X100, 0.2\% SDS, $10 \mathrm{mM}$ Tris$\mathrm{HCl}, 50 \mathrm{mM}$ EDTA, $\mathrm{pH}$ 7.5] and vortexed vigorously for 
$1 \mathrm{~min}$ before spinning at $10,000 \times g$ for $3 \mathrm{~min}$. $1 \mu \mathrm{L}$ supernatant can be applied in standard PCR mix with the addition of $2 \mathrm{mg} / \mathrm{mL}$ bovine serum albumin (Sigma-Aldrich; A 9418). Genomic DNA of selected $F$. solani transformants was isolated from 2 day old mycelium grown in YPG cultures at $25^{\circ} \mathrm{C}$ shaking $150 \mathrm{rpm}$ using the DNeasy plant mini kit (Qiagen) and a series of PCR reactions were used to verify that the cassette had been integrated correctly into the genome.

High-molecular weight genomic DNA was extracted from $50 \mathrm{mg}$ freeze-dried mycelium grown in $100 \mathrm{~mL}$ YPGmedium in 2 days at $25^{\circ} \mathrm{C}$. Extraction was preformed using DNeasy powersoil kit (QIAGEN), with following modifications: samples were homogenized for $1 \mathrm{~min}$ on a vortex adaptor instead of $10 \mathrm{~min}$ and all "vortex to mix" steps were excluded, and mixing was done by invert the tubes instead. The high molecular weight genomic DNA was prepared for sequencing using the 1D Native barcoding genomic DNA kit (EXP-NBD104 and SQK-LSK109) (Oxford Nanopore Technologies, Oxford, UK) and the sequencing was performed on a FLO-MINI106D (R9.4) flow-cell (Oxford Nanopore Technologies, Oxford, UK) with approximately 40-300 $\times$ coverage. Base-calling was preformed using Guppy v. 2.1.3 and demultiplexing and adaptor trimming was preformed using Porechop v. 0.2.3. The adaptor trimmed reads was filtered using Filtlong, reads less than 10.000 bp and with a mean quality score under 80 were excluded. Mapping of reads to the corresponding mutant reference genome was preformed using Minimap2.

\section{Quantification of fluorescence in the OE::eYFP transformant}

One of the verified transformants (OE::eYFP-6) was subjected to functional analyses. The strain was grown on $\mathrm{Cz}$ agar together with the wild type and analyzed via microscope. Pictures were taken at 10X magnification on an Olympus IX83 confocal quipped with a Yokogawa CSUW1 spinning disk and a Hamamatsu ORCA-Flash 4.0 camera. EFI projections were made from Z-stacks with 1.99 um Z-spacing (20- 48 frames) at $508 \mathrm{~nm}$ emission and $489 \mathrm{~nm}$ excitation wavelengths. Furthermore, The strain was grown together with the wild type at $25^{\circ} \mathrm{C}$ in 24 well polypropylene plates containing $1 \mathrm{~mL}$ of yeast mold (YM) medium, malt extract (ME) medium, potato dextrose (PD) medium, yeast extract sucrose (YES) medium, YPD medium, $(\mathrm{Cz})$ medium, $\mathrm{Cz}$ with yeast extract (CzY), $\mathrm{Cz}$ with $1 \mathrm{M} \mathrm{NaCl}$ and $\mathrm{Cz}$ with $\mathrm{NaNO}_{3}$ replaced with $\left(\mathrm{NH}_{4}\right)_{2} \mathrm{SO}_{4}$ [26]. Growth and fluorescence of both strains in four replicates were measured daily using an Infinite m1000pro (Tecan, Männeforf, Switzerland). Optical density (600 nm) was used to quantify growth and excitation/emission wavelength of 514/526 was used to quantify eYFP.
Generation of a fsr6 overexpression F. solani transformant Identification of the $f s r 6$ gene was done by BLASTx comparisons of known Fusarium fusarubin (PKS3) cluster transcription factor sequences $F$. fujikuroi fsr6 (FFUJ_03989, [38]) and F. graminearum pglR (FGSG_09188, [39]) towards the F. solani genome. We identified a 1167 bp gene, positions 251,539-252,705 on chromosome 10 (Additional file 2), which was recognized as an open reading frame on the reverse strand by GENSCAN [40] with the predicted function of a $\mathrm{Zn}(\mathrm{II})_{2} \mathrm{Cys}_{6}$ fungal transcription factor. To overexpress the fusarubin gene cluster in $F$. solani we chose a strategy were $e Y F P$ was substituted by the internal regulator $f s r 6$. Initially, eYFP was removed from the pSHUT4-eYFP vector using $B a m H I$ and $X h o I$ digestion. The digest was run on a $1 \%$ agarose gel and the linearized pSHUT4 backbone was purified with the QIAquick Gel Extraction Kit (Qiagen). The $f s r 6$ gene was amplified by PCR using the primers D100+E001, which each contained 20 bp overlaps to the linearized pSHUT4 backbone. The fsr6 gene and the pSHUT4 backbone were assembled in S. cerevisiae yeast recombinational assembly as described above. The verified pSHUT4-fsr6 vector was transformed into $F$. solani macroconidia as described above. The transformation yielded a single transformant, which was verified by colony PCR, short-read sequencing (Additional file 6) and whole genome sequencing as described above.

\section{Determination of growth and pigment production in the OE::fsr6 transformant}

The radial growth of the OE::fsr6 mutant was initially measured and compared to the parental $F$. solani wild type strain. The experiment was performed on solid $\mathrm{Cz}$ and YES medium in $90 \mathrm{~mm}$ petri dishes, which were inoculated with 1000 spores (resuspended in $0.2 \%$ agar) at the center of each plate using three replicates of each strain. The plates were incubated at $28{ }^{\circ} \mathrm{C}$ with daily growth measurements for 5 days where a cardinal axis was drawn from the inoculation point to facilitate the measurements in $\mathrm{X}$ and $\mathrm{Y}$ radii lengths. The colony area was estimated as Area $=r_{X} \cdot r_{Y} \cdot \pi$.

To compare pigment production in the OE: $f$ sr6 strain to the wild type $F$. solani strain, 5000 spores were added to $50 \mathrm{~mL}$ YES medium in $250 \mathrm{~mL}$ baffled flasks in triplicates. The flasks were incubated for 6 days at $28{ }^{\circ} \mathrm{C}$ and $100 \mathrm{rpm}$. The produced pigments in the broth was extracted using a mixture of chloroform and methanol as previously described [28]. The chloroform phase was evaporated to dryness on a rotary evaporator at $40^{\circ} \mathrm{C}$ and resuspended in $1 \mathrm{~mL}$ DMSO. The extracts were analyzed on a Hitachi Elite LaChrom HPLC system equipped with a $150 \times 4.6 \mathrm{~mm}$ Ascentis Xpress $2.7 \mathrm{~mm}$ phenyl-hexyl column (Sigma Aldrich, USA) and coupled to a high 
resolution mass spectrometer (compact qTOF, Bruker, Germany) operating in positive ionization mode using the settings as previously described [41].

To verify the identity of bostrycoidin and javanicin, twenty $0.6 \mathrm{~mm}$ plugs from the OE::fsr6 transformant were used to inoculate $2 \times 1 \mathrm{~L} \mathrm{YES} \mathrm{medium.} \mathrm{The} \mathrm{culture}$ was grown for 7 days at $25{ }^{\circ} \mathrm{C}$ and $105 \mathrm{rpm}$, before the medium was extracted as described above. The resulting extract was subjected to purification by a preparative HPLC (Waters HPLC pump 515; Waters Binary Gradient Module 2545; Waters System Fluidics Organizer) coupled to a quadrupole mass analyzer (Waters Acquity QDa), a UV/Vis detector (Waters UV/Visible detector 2489) and a fraction collector (Waters Sample Manager 2767). The sample was separated by a C6-phenyl column (Phenomenex Gemini $5 \mu \mathrm{m}$ C6-Phenyl, $110 \AA$, $250 \times 10 \mathrm{~mm}$ ) by injecting $1 \mathrm{~mL}$ per run and applying a linear gradient of water (VWR, HiPerSolv CHROMANORM HPLCMS grade) and acetonitrile (VWR, HiPerSolv CHROMANORM HPLC-MS grade), both supplemented with $0.1 \%$ formic acid (VWR, 99\% HiPerSolv CHROMANORM LC-MS grade). The gradient initiated at 10\% acetonitrile, increased to $99 \%$ over $15 \mathrm{~min}$, and held at $99 \%$ for $5 \mathrm{~min}$ with a constant flow at $10 \mathrm{~mL} / \mathrm{min}$. The system was controlled by MassLynx V4.2 set to collect the masses $M=290.1 \mathrm{Da}$ and $\mathrm{M}=285.1 \mathrm{Da}$. Javanicin and bostrycoidin eluted after 9.3 and $10.0 \mathrm{~min}$, respectively. The collected fractions were lyophilized and resuspended in $\mathrm{CDCl}_{3}$ (euroiso-top, $0.03 \%$ tetramethylsilane (TMS)) and ${ }^{1} \mathrm{H}$ nuclear magnetic spectroscopy (NMR) spectra were recorded on a Bruker AVIII-600 MHz spectrometer (Bruker, Karlsruhe, Germany) equipped with a triple resonance cryogenically cooled probe with z-gradients and controlled by TopSpin 3.5pl6. All spectra were recorded at $298.1 \mathrm{~K}$ and calibrated to internal TMS. The obtained spectra were compared to published data for javanicin and bostrycoidin $[42,43]$.

\section{Results and discussion}

\section{Assembly of shuttle vector pSHUT4::eYFP by yeast} mediated recombinational cloning

To generate pSHUT4-eYFP four PCRs were performed to amplify the backbone, expression cassette and the two integration border sites using primers carrying
15 bp homology to the neighboring fragment (30 bp total homology between all fragment pairs). The resulting products were visualized by gel electrophoresis, which matched the expected sizes (Fig. 1). The four fragments were transformed into $S$. cerevisiae and six resulting colonies were selected to verify that the plasmid had been correctly assembled. After propagation in E. coli, the plasmids were digested with PstI and BglII, which resulted in the anticipated band pattern. Three plasmids were validated further by PCR yielding identical and correct band lengths. The eYFP cassette was ultimately sequenced in one of the plasmids, which confirmed that the plasmid had been correctly assembled (Additional file 3).

\section{Fungal transformation and validation}

The verified pSHUT4:eYFP was transformed into $F$. solani by ATMT, which resulted in more than 30 fungal colonies from 10 plates, equal to 25 fungal colonies per $10^{7}$ conidia. Seven randomly picked colonies were streaked on selective PDA of which six showed uninhibited growth. Subsequent diagnostic colony PCR showed furthermore that the T-DNA had correctly recombined into the integration site in all colonies tested (Primer positions marked in Fig. 2a). Two of the transformants displaying fluorescence (Fig. 2b), OE::eYFP-2 and -6 , were selected for further validation using a PCR based strategy. The initial PCR confirmed that the transformants contain the nptII gene. The following PCR with primers D096+D097 yielded only a product in the wild type, possibly because the theoretical size of $5983 \mathrm{bp}$ in the transformants was too large for successful amplification. This suggests that the T-DNA had been inserted into the correct locus, which was further verified when primers targeting the T-DNA were combined in PCRs with primers targeting the up- and downstream regions (Additional file 4). Following a short-read sequencing of the final two PCR products validating correct recombination, we performed whole genome sequencing of the mutant ultimately verifying correct integration (Fig. 2c).

Functional characterization of $F$. solani eYFP transformants

To determine that EYFP was functionally expressed by the TEF-1 $\alpha$ promoter from the integration site, OE::eYFP-6

(See figure on next page.)

Fig. 2 Construction and validation of eYFP overexpression mutant FS OE::eYFP-6. a Integration of pSHUT4-eYFPT-DNA cassette into a non-coding position of the F. solani genome through homologous recombination between identical segments (blue). Primer positions are marked, and a full list of primers is found in Additional file 1. b DIC pictures and EFI projections comparing parental and mutant strain phenotypes. Scale bar $=100 \mu \mathrm{m}$. $\mathbf{c}$ Genome sequencing validation displays correct integration of the PSHUT4-eYFPT-DNA cassette via homologous recombination. $\mathbf{d}$ Growth and eYFP induction of F. solani wild type and OE::eYFP-6 during growth on nine different media. The left panel illustrates growth (optical density) over a 7 days period. The right panel illustrates fluorescence at excitation/emission wavelength of 514/526. All data points are the average of four biological replicates, expressed as a percentage of the maximum observed value 
a

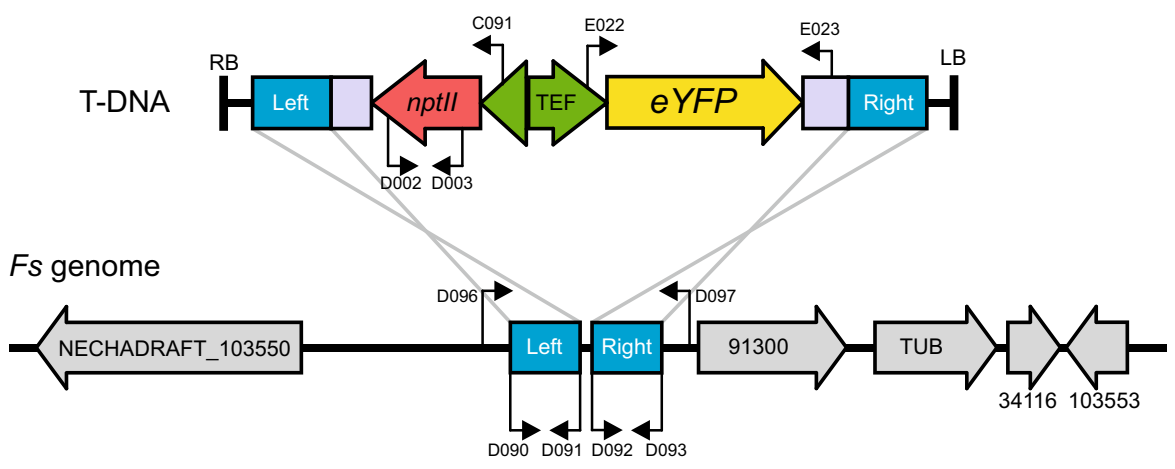

b

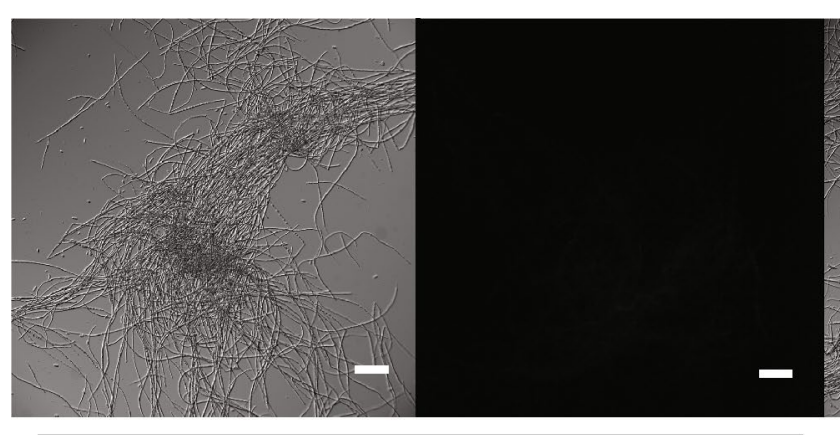

Fs WT

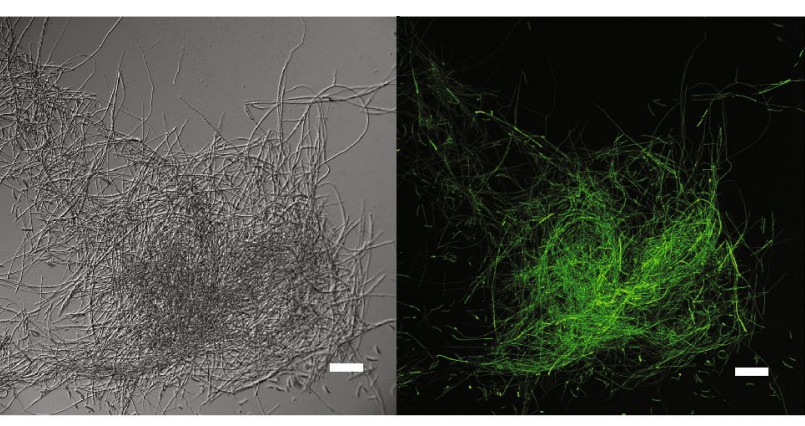

FsOE::eYFP

C

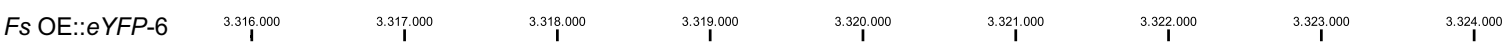

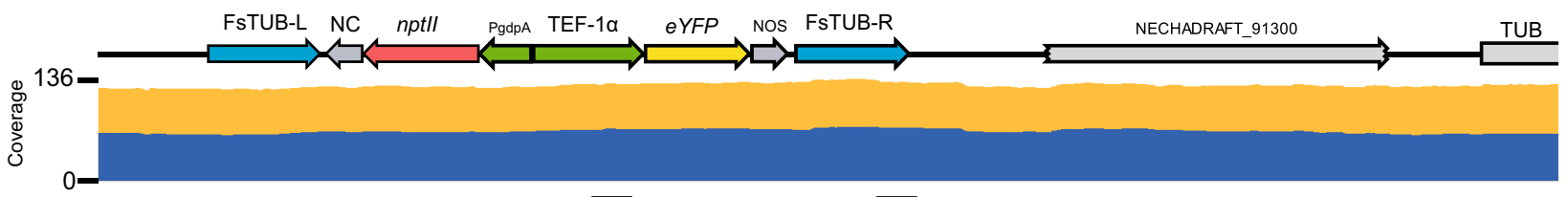

Forward reads $\square$ Reverse reads
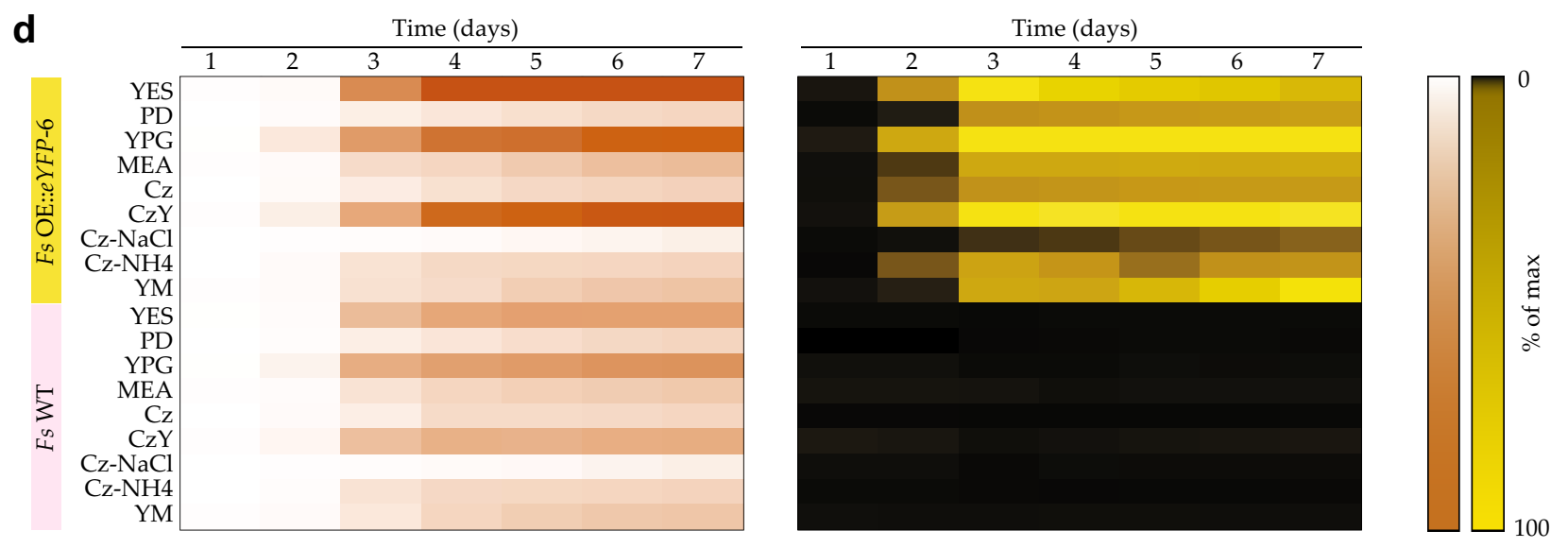
was selected for further analyses in a time study together with the wild type. The results showed that fluorescence could be detected after 2 days on all media, except for PDA and $\mathrm{Cz}-\mathrm{NaCl}$, which was delayed by 1 day (Fig. $2 \mathrm{~d}$ ). The fluorescence signal continued throughout the experiment on all media, although a decrease followed by stagnation was observed on YES, YPG and Cz. This could indicate the cultures had reached a phase with reduced cellular activity caused by nutrient depletion. The observation that the promoter is active on all the media tested is valuable information in future studies where the examined target genes require a specific growth medium or condition.

\section{Targeted activation of the PKS3 gene cluster results} in production of javanicin and bostrycoidin pigments

We identified an orthologue to the local transcription factor $f s r 6$ controlling activation of the PKS3 biosynthetic gene cluster in F. fujikuroi (Fig. 3a). The gene was rapidly replaced in the Agrobacterium expression cassette of pSHUT4-eYFP yielding the plasmid pSHUT4-fsr6 (for plasmid validation, see Additional file 5) (Fig. 3b). The fungal transformation yielded a single mutant with a distinct deep red/purple phenotype, which is visibly different from the white color of the wild type when grown on PDA medium (Fig. 3c). In addition to the $f s r 6$ gene, we applied the presented system to introduce and express several other genes in F. solani (not shown). In our experience, the system, on average, yields one transformant per plate. During the isolation process of the OE::fsr6 transformant, we observed a reduced growth rate. The transformant was therefore grown on $\mathrm{Cz}$ and YES agar medium together with the wild type. This experiment confirmed that the transformant grew significantly slower than wild type (Fig. 3d). This reduced growth rate could be due to the accumulation of pigments and thereby be responsible for the low transformation success. The transformant was subsequently screened by diagnostic PCR, which indicated that the expression cassette had been correctly inserted (Additional file 6). This was further validated by whole genome sequencing of Fs OE::fsr6 (Fig. 3e). Analysis on HPLC-HRMS of the secondary metabolites produced in liquid cultures of and wild type F. solani strain and the OE::fsr6 displayed two dominant peaks not visible in wild type extracts at 7.5 and 8.2 min with masses matching javanicin and bostrycoidin (Fig. 3f). These compounds were subsequently isolated by preparative HPLC and their identity confirmed by NMR (Additional files 7 and 8). These two compounds are derived from the PKS3 cluster and thereby confirming functional integration of the $f s r 6$ overexpression cassette into F. solani.

\section{Future potential of the vector system}

The aim of our study was to develop a platform for reliable targeted transformation of $F$. solani, which can be used to increase our understanding on this important pathogenic fungus. One research area that can benefit from the platform is the secondary metabolite research in Fusarium. So far, the only secondary metabolite to be linked to a gene directly in F. solani is sansalvamide [21]. However, comparative analyses have revealed that F. solani contain numerous biosynthetic gene clusters, including 13 polyketide synthases $(P K S s)$ of which only three can be linked to a product based on orthology $[12-14,16,17,44]$. The pSHUT4-eYFP vector can be modified and used to overexpress local transcription factors of biosynthetic gene clusters, which has proven a highly successful approach to identify novel secondary metabolites [45]. This approach has already been adapted in other Fusarium species, which for example has led to discovery of fusarielins in F. graminearum [46], equisetin in F. heterosporum [47] and fujikurins in F. fujikuroi [48]. The simple workflow needed to overexpress a target gene (e.g. transcription factor) is illustrated in Fig. $2 \mathrm{~b}$. First, the $e Y F P$ has to be looped out by digesting the plasmid with XhoI and BamHI. The target gene can then be inserted into the linearized plasmid by yeast recombinational cloning and transferred into an active position in the F. solani genome by ATMT.

Among the 13 PKSs in F. solani, we have identified putative transcription factors for seven gene clusters for which we have adopted this system. Other fungal species can however also be targeted in a similar approach, the only requirement is that guiding integration border sites should be replaced with a suiting region from the target organism. Potentially, the presented vector could be used to transform closely related members of the FSSC given the integration locus shares high sequence similarity to the integration site in F. solani f. sp. pisi. A similar locus in the genome of $F$. neocosmosporiellum shares $86 \%$ nucleotide content with the integration border sites [49], however, whether pSHUT4 can successfully be applied in such a transformation experiment remains to be tested. It should be noted by researchers wishing to apply the system for genetic studies concerning signal transduction or virulence etc., we recommend carefully monitoring potential change the expression of neighboring genes when introducing any expression cassette, as introduction of recombinant DNA may compromise the intrinsic regulation. The system is not restricted to ATMT as linearized or circular plasmids can be used for protoplast transformation in microorganisms where this is feasible. 

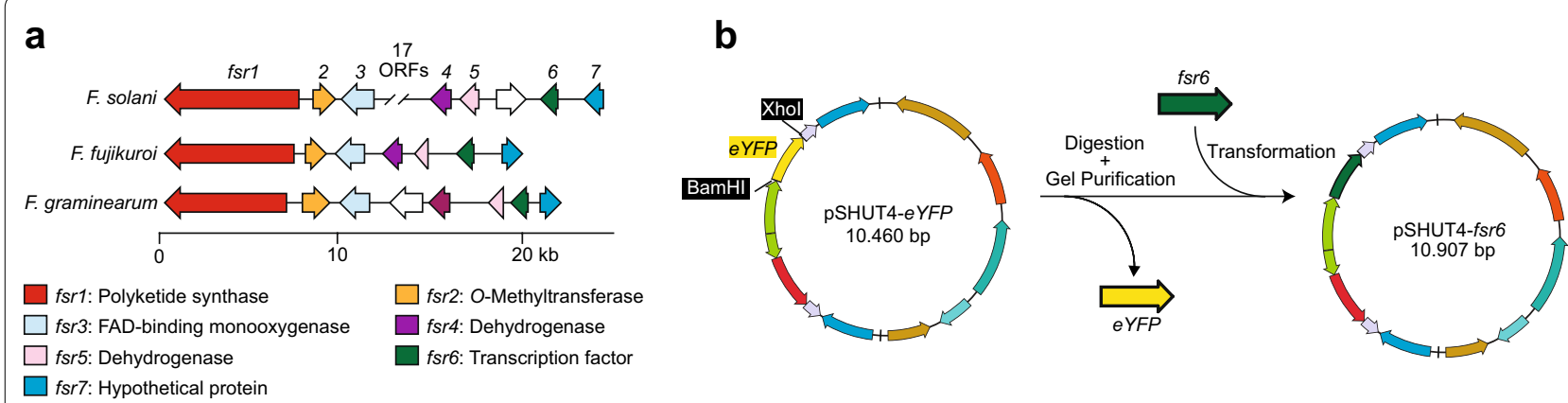

C

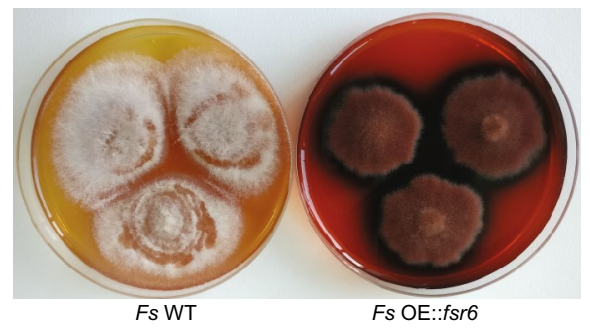

d
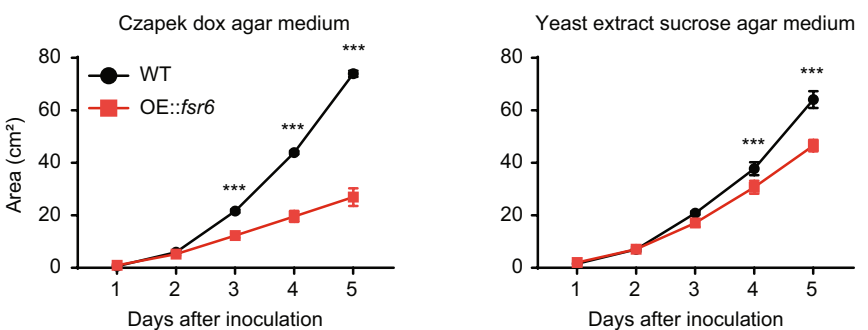

e

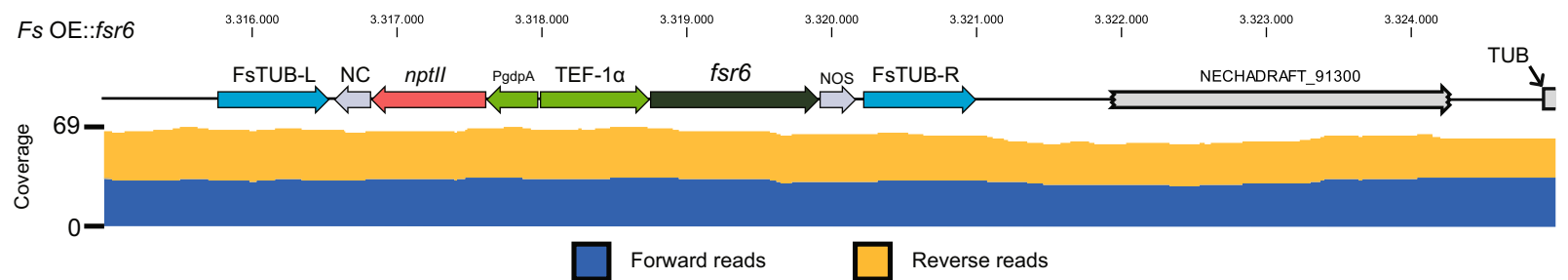

f

Bostrycoidin

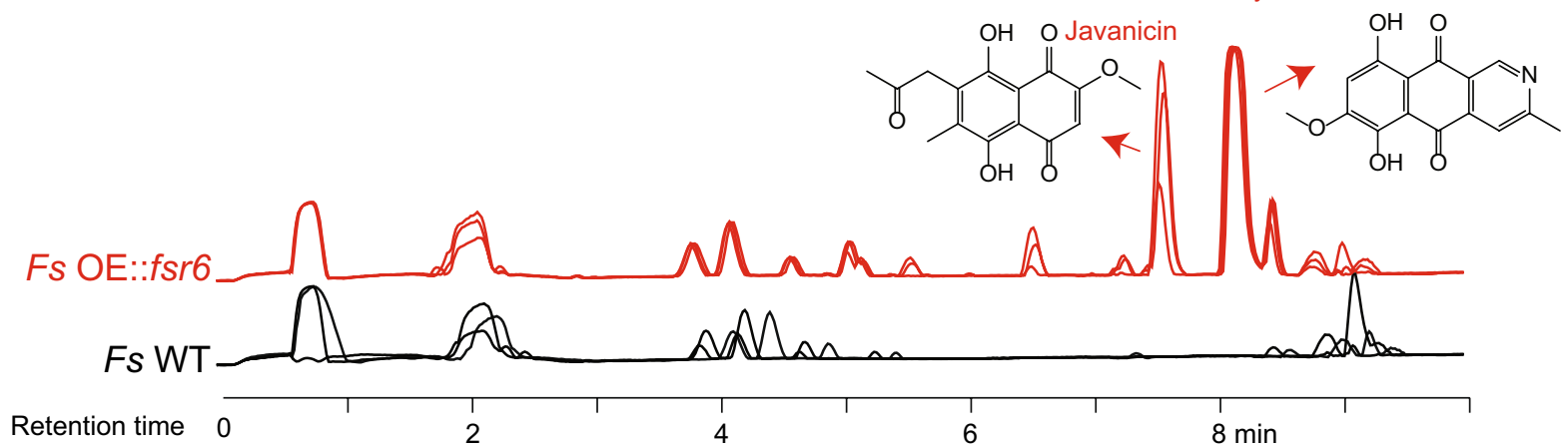

Fig. 3 Construction and validation of overexpression mutant Fs OE:.fsr6. a Illustration of fsr gene cluster orthologues in Fusarium solani, F. fujikuroi and F. graminearum. b Workflow for replacement of the eYFP gene for fsr6 in the agrobacterium shuttle vector pSHUT4 via yeast mediated recombinational assembly. c Photographs of $F$. solani wild type and fsr6 overexpression mutant. $\mathbf{d}$ growth diameter test on Czapek dox and yeast sucrose agar media. e genome sequencing of Fs OE::fsr6 reveals correct integration of the pSHUT4-fsr6 T-DNA cassette in the locus via homologous recombination. $\mathbf{f H P L C}-\mathrm{MS}$ total ion chromatograms for metabolite extracts from F. solani wild type and the fsr6 overexpression mutant in triplicates. The compounds javanicin $[\mathrm{M}+\mathrm{H}]^{+}=291.0868$ and bostrycoidin $[\mathrm{M}+\mathrm{H}]^{+}=286.0715$ were observed in the mutant extracts

\section{Conclusions}

In this study we present the implementation of a novel vector system that allows for fungal transformation and strong gene expression in the crop-pathogen F. solani. As a case study, we performed targeted activation of the biosynthetic $f s r$ gene cluster responsible for mycelial pigmentation. From cultivation experiments in this study, we were able to isolate and confirm the chemical structures of the red pigments javanicin and bostrycoidin. Targeted overexpression of transcriptional regulators is thus 
a feasible approach to activate biosynthetic gene clusters as a venue for isolating and describing novel secondary metabolites in the FSSC.

\section{Supplementary information}

Supplementary information accompanies this paper at https://doi. org/10.1186/s40694-019-0089-2.

\author{
Additional file 1. List of primers. \\ Additional file 2. fsr6 nucleotide sequence. \\ Additional file 3. PSHUT4-eYFP plasmid validation. \\ Additional file 4. F. solani OE::. YFP mutant colony PCR validation.
}

Additional file 5. pSHUT4-fsr6 plasmid validation.

Additional file 6. F. solani OE::fsr6 mutant validation by PCR and TubeSeq sequencing.

Additional file 7. NMR table of javanicin isolated from Fs OE::fsr6.

Additional file 8. NMR table of bostrycoidin isolated from Fs OE::fsr6.

\section{Abbreviations}

ATMT: Agrobacterium tumefaciens-mediated transformation; CMC: carboxymethyl-cellulose; Cz: Czapek Dox; CzY: Czapek Dox-Yeast extract; DIC: differential interference contrast microscopy; EFI: extended focal image; eYFP: enhanced yellow fluorescent protein; Fs: Fusarium solani; FSSC: Fusarium solani species complex; HPLC-HRMS: high-pressure liquid chromatography-High resolution mass spectrometry; ME: malt extract; OE: overexpression; PCR: polymerase chain reaction; PD: potato dextrose; PKS: polyketide synthase; qTOF: quadropole time-of-flight; SC-U: yeast synthetic dropout medium without uracil; T-DNA: transfer-DNA; YES: yeast extract-sucrose; YM: yeast mold; YPG: yeast extract-peptone-glucose.

\section{Acknowledgements}

Not applicable.

\section{Authors' contributions}

MRN, TES and JLS designed the experiments. MRN, AKRH and KK constructed all plasmids and performed genetic manipulations. TS performed whole genome sequencing and data analysis. EB, NC and SEKK carried out the growth and cultivation experiments. MRN and KRW extracted metabolites, and RW was responsible for metabolite analysis. TS and TES conducted microscopy. MRN, RW, TES and JLS interpreted data and results. MRN was the major contributor in writing the manuscript, while JLS provided critical feedback. All authors read and approved the final manuscript.

\section{Funding}

The study was supported by the Novo Nordisk Foundation

(NNF18OC0034952, NNF15OC0016186 and NNF15OC0016028) and the Obel Family Foundation.

\section{Availability of data and materials}

The whole genome sequencing datasets analyzed during the current study are available from the corresponding author on reasonable request. The sequences of primers included in this article can be found in Additional file 1. The PSHUT4-eYFP plasmid is available at the Addgene repository (\# 133094).

\section{Ethics approval and consent to participate}

Not applicable.

\section{Consent for publication \\ Not applicable.}

\section{Competing interests}

The authors declare that they have no competing interests.

\section{Author details}

${ }^{1}$ Department of Chemistry and Bioscience, Aalborg University, Niels Bohrs Vej 8, 6700 Esbjerg, Denmark. ${ }^{2}$ Department of Chemistry and Bioscience, Aalborg University, Fredrik Bajers Vej 7H, 9220 Aalborg Ø, Denmark.

Received: 29 September 2019 Accepted: 25 November 2019 Published online: 11 December 2019

\section{References}

1. Zhang N, O'Donnell K, Sutton DA, Nalim FA, Summerbell RC, Padhye AA, Geiser DM. Members of the Fusarium solani species complex that cause infections in both humans and plants are common in the environment. J Clin Microbiol. 2006;44(6):2186-90.

2. Coleman JJ. The Fusarium solani species complex: ubiquitous pathogens of agricultural importance. Mol Plant Pathol. 2016;17(2):146-58.

3. O'Donnell K. Molecular phylogeny of the Nectria haematococca-Fusarium solani species complex. Mycologia. 2000;92(5):919-38.

4. O'Donnell K, Sutton DA, Fothergill A, McCarthy D, Rinaldi MG, Brandt ME, Zhang N, Geiser DM. Molecular phylogenetic diversity, multilocus haplotype nomenclature, and in vitro antifungal resistance within the Fusarium solani species complex. J Clin Microbiol. 2008;46(8):2477-90.

5. Šišić A, Al-Hatmi AMS, Baćanović-Šišić J, Ahmed SA, Dennenmoser D, de Hoog GS, Finckh MR. Two new species of the Fusarium solani species complex isolated from compost and hibiscus (Hibiscus sp.). Antonie Van Leeuwenhoek. 2018;111(10):1785-805.

6. Kolattukudy PE, Gamble DL. Nectria haematococca: pathogenesis and host specificity in plant diseases. In: Kohmoto K, Singh US, Singh RP, editors. Pathogenesis and host specificity in plant pathogenic fungi and nematodes. Oxford: Pergamon; 1995. p. 83-102.

7. Bueno CJ, Fischer IH, Rosa DD, Firmino AC, Harakava R, Oliveira CMG, Furtado EL. Fusarium solani f. sp passiflorae: a new forma specialis causing collar rot in yellow passion fruit. Plant Pathol. 2014;63(2):382-9.

8. Chung WC, Chen LW, Huang JH, Huang HC, Chung WH. A new'forma specialis' of Fusarium solani causing leaf yellowing of Phalaenopsis. Plant Pathol. 2011;60(2):244-52.

9. Suga H, Ikeda S, Taga M, Kageyama K, Hyakumachi M. Electrophoretic karyotyping and gene mapping of seven formae speciales in Fusarium solani. Curr Genet. 2002;41(4):254-60.

10. Šišić A, Baćanović-Šišić J, Al-Hatmi AMS, Karlovsky P, Ahmed SA, Maier W, de Hoog GS, Finckh MR. The 'forma specialis' issue in Fusarium: a case study in Fusarium solani f. sp. pisi. Sci Rep. 2018;8(1):1252.

11. Short DP, O'Donnell K, Thrane U, Nielsen KF, Zhang N, Juba JH, Geiser DM. Phylogenetic relationships among members of the Fusarium solani species complex in human infections and the descriptions of $F$. keratoplasticum sp. nov. and F. petroliphilum stat. nov. Fungal Genet Biol. 2013;53:59-70.

12. Hansen FT, Gardiner DM, Lysøe E, Fuertes PR, Tudzynski B, Wiemann P, Sondergaard TE, Giese H, Brodersen DE, Sørensen JL. An update to polyketide synthase and non-ribosomal synthetase genes and nomenclature in Fusarium. Fungal Genet Biol. 2015;75:20-9.

13. Hansen FT, Sørensen JL, Giese H, Sondergaard TE, Frandsen RJ. Quick guide to polyketide synthase and nonribosomal synthetase genes in Fusarium. Int J Food Microbiol. 2012;155(3):128-36.

14. Janevska S, Arndt B, Niehaus EM, Burkhardt I, Rosler SM, Brock NL, Humpf HU, Dickschat JS, Tudzynski B. Gibepyrone biosynthesis in the rice pathogen Fusarium fujikuroi is facilitated by a small polyketide synthase gene cluster. J Biol Chem. 2016;291(53):27403-20.

15. Brown DW, Butchko RAE, Baker SE, Proctor RH. Phylogenomic and functional domain analysis of polyketide synthases in Fusarium. Fungal Biology. 2012;116(2):318-31.

16. Brown DW, Butchko RAE, Busman M, Proctor RH. Identification of gene clusters associated with fusaric acid, fusarin, and perithecial pigment production in Fusarium verticillioides. Fungal Genet Biol. 2012;49(7):521-32.

17. Nielsen MR, Sondergaard TE, Giese H, Sørensen JL. Advances in linking polyketides and non-ribosomal peptides to their biosynthetic gene clusters in Fusarium. Curr Genet. 2019. https://doi.org/10.1007/s0029 4-019-00998-4.

18. Sang H, Witte A, Jacobs JL, Chang HX, Wang J, Roth MG, Chilvers MI. Fluopyram sensitivity and functional characterization of $S d h B$ in the 
Fusarium solani species complex causing soybean sudden death syndrome. Front Microbiol. 2018;9:2335.

19. Liu H, Bao X. Overexpression of the chitosanase gene in Fusarium solani via Agrobacterium tumefaciens-mediated transformation. Curr Microbiol. 2009;58(3):279-82.

20. Sandrock RW, Vanetten HD. The relevance of tomatinase activity in pathogens of tomato: disruption of the beta(2)-tomatinase gene in Colletotrichum coccodes and Septoria lycopersici and heterologous expression of the Septoria lycopersici beta(2)-tomatinase in Nectria haematococca, a pathogen of tomato fruit. Physiol Mol Plant Pathol. 2001;58(4):159-71.

21. Romans-Fuertes $P$, Sondergaard TE, Sandmann MI, Wollenberg RD, Nielsen KF, Hansen FT, Giese H, Brodersen DE, Sørensen JL. Identification of the non-ribosomal peptide synthetase responsible for biosynthesis of the potential anti-cancer drug sansalvamide in Fusarium solani. Curr Genet. 2016;62:799-807.

22. Frandsen RJN. A guide to binary vectors and strategies for targeted genome modification in fungi using Agrobacterium tumefaciens-mediated transformation. J Microbiol Methods. 2011;87(3):247-62.

23. Kevin RO, Vo KT, Michaelis S, Paddon C. Recombination-mediated PCR-directed plasmid construction in vivo in yeast. Nucleic Acids Res. 1997;25(2):451-2.

24. Joska TM, Mashruwala A, Boyd JM, Belden WJ. A universal cloning method based on yeast homologous recombination that is simple, efficient, and versatile. J Microbiol Methods. 2014;100:46-51.

25. Kieler JB, Duong KL, Moye-Rowley WS, Klutts JS. Targeted gene deletion in Aspergillus fumigatus using microbial machinery and a recyclable marker. J Microbiol Methods. 2013:95(3):373-8.

26. Frisvad J. Media and Growth Conditions for Induction of Secondary Metabolite Production. In: Keller NP, Turner G, editors. Fungal Secondary Metabolism, vol. 944. Totowa: Humana Press; 2012. p. 47-58.

27. Brachmann CB, Davies A, Cost GJ, Caputo E, Li J, Hieter P, Boeke JD. Designer deletion strains derived from Saccharomyces cerevisiae S288C: a useful set of strains and plasmids for PCR-mediated gene disruption and other applications. Yeast. 1998;14(2):115-32.

28. Westphal K, Wollenberg R, Herbst F-A, Sørensen J, Sondergaard T, Wimmer R. Enhancing the production of the fungal pigment aurofusarin in Fusarium graminearum. Toxins. 2018;10(11):485.

29. Earley KW, Haag JR, Pontes O, Opper K, Juehne T, Song K, Pikaard CS. Gateway-compatible vectors for plant functional genomics and proteomics. Plant J. 2006;45(4):616-29.

30. Lukassen MB, Saei W, Kumar A, Tamminen A, Sondergaard TE, Kempken F, Wiebe MG, Sørensen JL. Identification of the scopularide gene cluster in Scopulariopsis brevicaulis. Mar Drugs. 2015;13:4331-43.

31. Nielsen MR, Wollenberg RD, Westphal K, Sondergaard TE, Wimmer R, Gardiner DM, Sørensen JL. Heterologous expression of intact biosynthetic gene clusters in Fusarium graminearum. Fungal Genet Biol. 2019;132:103248.

32. Josefsen L, Droce A, Sondergaard TE, Sørensen JL, Bormann J, Schaefer W, Giese H, Olsson S. Autophagy provides nutrients for nonassimilating fungal structures and is necessary for plant colonization but not for infection in the necrotrophic plant pathogen Fusarium graminearum. Autophagy. 2012;8(3):326-37.

33. Sikorski RS, Hieter P. A system of shuttle vectors and yeast host strains designed for efficient manipulation of DNA in Saccharomyces cerevisiae. Genetics. 1989:122(1):19-27.

34. Gietz RD, Schiestl RH. High-efficiency yeast transformation using the LiAc/SS carrier DNA/PEG method. Nat Protoc. 2007;2(1):31-4.

35. Noskov VN, Lee NCO, Larionov V, Kouprina N. Rapid generation of long tandem DNA repeat arrays by homologous recombination in yeast to study their function in mammalian genomes. Biol Proc Online. 2011;13(1):8.

36. Cappellini RA, Peterson JL. Macroconidium formation in submerged cultures by a non-sporulating strain of Gibberella zeae. Mycologia. 1965;57(6):962-6
37. Simmons EG. Alternaria taxonomy: current status, viewpoints, challenge. In: Chelkowski J, Visconti A, editors. Alternaria: biology, plant diseases and metabolites. Amsterdam: Elsevier; 1992. p. 1-36.

38. Studt L, Wiemann P, Kleigrewe K, Humpf H-U, Tudzynski B. Biosynthesis of fusarubins accounts for pigmentation of Fusarium fujikuroi perithecia. Appl Environ Microbiol. 2012;78(12):4468-80.

39. Frandsen RJN, Rasmussen SA, Knudsen PB, Uhlig S, Petersen D, Lysøe E, Gotfredsen CH, Giese H, Larsen TO. Black perithecial pigmentation in Fusarium species is due to the accumulation of 5-deoxybostrycoidinbased melanin. Scientific Reports. 2016;6:26206.

40. Burge C, Karlin S. Prediction of complete gene structures in human genomic DNA. J Mol Biol. 1997;268(1):78-94.

41. Wollenberg RD, Sondergaard TE, Nielsen MR, Knutsson S, Pedersen TB, Westphal KR, Wimmer R, Gardiner DM, Sørensen JL. There it is! Fusarium pseudograminearum did not lose the fusaristatin gene cluster after all. Fungal Biology. 2018;123:10-7.

42. Chowdhury NS, Sohrab MH, Rana MS, Hasan CM, Jamshidi S, Rahman KM. Cytotoxic naphthoquinone and azaanthraquinone derivatives from an endophytic fusarium solani. J Nat Prod. 2017;80(4):1173-7.

43. Yamamoto Y, Kinoshita Y, Ran Thor G, Hasumi M, Kinoshita K, Koyama K, Takahashi K, Yoshimura I. Isofuranonaphthoquinone derivatives from cultures of the lichen Arthonia cinnabarina (DC.) Wallr. Phytochemistry. 2002;60(7):741-5.

44. Brown DW, Proctor RH. Insights into natural products biosynthesis from analysis of 490 polyketide synthases from Fusarium. Fungal Genet Biol. 2016;89:37-51.

45. Bergmann S, Schumann J, Scherlach K, Lange C, Brakhage AA, Hertweck C. Genomics-driven discovery of PKS-NRPS hybrid metabolites from Aspergillus nidulans. Nat Chem Biol. 2007;3(4):213-7.

46. Sørensen JL, Hansen FT, Sondergaard TE, Staerk D, Lee TV, Wimmer R, Klitgaard LG, Purup S, Giese H, Frandsen RJ. Production of novel fusarielins by ectopic activation of the polyketide synthase 9 cluster in Fusarium graminearum. Environ Microbiol. 2012;14(5):1159-70.

47. Kakule TB, Sardar D, Lin ZJ, Schmidt EW. Two related pyrrolidinedione synthetase loci in Fusarium heterosporum ATCC 74349 produce divergent metabolites. ACS Chem Biol. 2013;8(7):1549-57.

48. von Bargen KW, Niehaus EM, Krug I, Bergander K, Wurthwein EU, Tudzynski B, Humpf HU. Isolation and structure elucidation of fujikurins A-D: products of the PKS19 gene cluster in Fusarium fujikuroi. J Nat Prod. 2015;78(8):1809-15

49. Kim W, Cavinder B, Proctor RH, O'Donnell K, Townsend JP, Trail F. Comparative genomics and transcriptomics during sexual development gives insight into the life history of the cosmopolitan fungus Fusarium neocosmosporiellum. Frontiers in Microbiology. 2019. https://doi.org/10.3389/ fmicb.2019.01247.

\section{Publisher's Note}

Springer Nature remains neutral with regard to jurisdictional claims in published maps and institutional affiliations.

Ready to submit your research? Choose BMC and benefit from:

- fast, convenient online submission

- thorough peer review by experienced researchers in your field

- rapid publication on acceptance

- support for research data, including large and complex data types

- gold Open Access which fosters wider collaboration and increased citations

- maximum visibility for your research: over $100 \mathrm{M}$ website views per year

At BMC, research is always in progress.

Learn more biomedcentral.com/submissions 